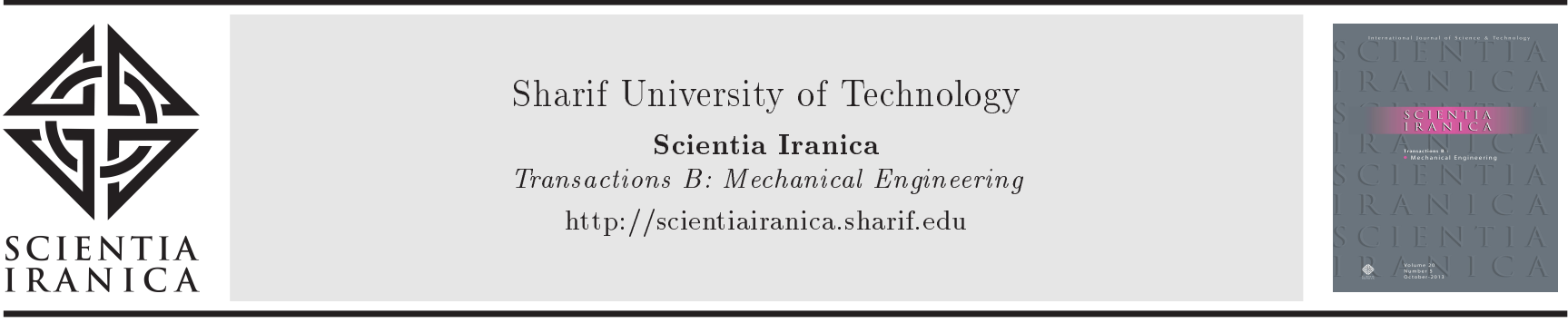

Research Note

\title{
Dispersion of Stoneley waves through the irregular common interface of two hydrostatic stressed MTI media
}

\author{
S. Chowdhury ${ }^{a}$, S. Kundu ${ }^{a}$, P. Alam ${ }^{b, *}$, and Sh. Gupta ${ }^{a}$ \\ a. Department of Mathematics and Computing, Indian Institute of Technology (Indian School of Mines), Dhanbad-826004, \\ Jharkhand, India. \\ b. Department of Mathematics, School of Advanced Sciences, Vellore Institute of Technology, Vellore-632014, Tamil Nadu, India.
}

Received 9 January 2019; received in revised form 4 January 2020; accepted 1 February 2020

\author{
KEYWORDS \\ Stoneley wave; \\ Magneto-elasticity; \\ Hydrostatic stress; \\ Transversely isotropic; \\ Corrugation; \\ Maxwell's equations.
}

\begin{abstract}
The present study investigates the mathematical inspection of Stoneley wave propagation through the corrugated irregular common interface of two dissimilar Magnetoelastic Transversely Isotropic (MTI) half-space media under the impression of hydrostatic stresses. To enumerate the Lorentz's force besmeared in the structure, the generalized Ohm's law and Maxwell's equations were considered. The interior deformations were analytically calculated to obtain the wave frequency equation through the prescribed boundary conditions. To investigate the impacts of irregularity and various affecting parameters such as magnetic couplings and hydrostatic stresses on the wave propagation, frequency curves formed for the phase velocity of the wave.
\end{abstract}

(C) 2021 Sharif University of Technology. All rights reserved.

\section{Introduction}

Earth is a bizarre collection of elastic media. To collect enough information on the composition of the interior of the Earth, seismic body waves were carefully checked. In addition, for theoretical inspection, one must take into account a number of appropriate models. These models provide indirect data on the internal structure of the Earth that can help seismologists and geophysicists figure out different physical properties, state, and configuration of the interior of the Earth's

\footnotetext{
*. Corresponding author.

E-mail addresses: presisoumitra.math@gmail.com (S. Chowdhury); kundu_santi@yahoo.co.in (S.Kundu); alamparvez.amu@gmail.com (P.Alam); shishir_ism@yahoo.com (Sh. Gupta).
}

doi: $10.24200 /$ sci. 2020.52653 .2820 medium and explore the valuable and unknown materials beneath.

Stoneley [1] demonstrated the conceivable presence of another type of waves (named after him as Stoneley waves) that were identical to surface waves propagating through interfaces of either solid and liquid media or two elastic media and found a secular relation for the wave; he pointed to the condition of existence of these kinds of waves. Variations of the propagation attributes of Stoneley waves in fluid-filled media were thoroughly examined by Ashour [2]. The dispersion equation for the Stoneley waves was introduced by Abo-Dahab [3] for magneto-thermoelastic materials. Tiwana et al. [4] investigated the consequences of spherical wave diffraction due to a point source from a Perfect Electromagnetic Conducting (PEMC) half plane.

The internal layer of the Earth comprises different types of substances with non-identical types of prop- 
erties including homogeneous, heterogeneous, transversely isotropic and orthotropic, etc. Transversely isotropic substances are a particular type of orthotropic substances symmetrical about an axis that is normal to a plane of isotropy. The hexagonal crystals exist in the collection of transversely isotropic solids. Investigations of the wave regulations in transversely isotropic and orthotropic (fiber-reinforced [5]) materials are of significance to the improvement of a number of principal studies on mechanics. Recently, Alam et al. [6] and Kundu et al. [7] studied surface waves in transversely isotropic layers. Plane wave propagation was examined by Singh [8] in a thermoelastic transversely isotropic half-space.

Realistically, the Earth surface is not a continuous and regular plane. The interfaces of two adjacent layers are irregular in nature and complicated. Irregular interfaces are found in many forms including corrugation (undulated), parabolic, rectangular, etc. This is the reason why considering the impact of irregularity while studying the propagation of Stoneley waves is of significance. A number of authors have investigated seismic waves through irregular media, among whom Vishwakarma and Xu [9], Singh [10], Alam et al. [11], and Saroj et al. [12] are notable.

The hydrostatic stress (confining stress) is an isotropic stress caused by the weight-induced pressure of water on a certain point of the medium. Hydrostatic stress has considerable applications in geophysics and geomechanics. As an isotropic stress, hydrostatic stress behaves uniformly in all directions. Addy and Chakraborty [13] examined the effects of hydrostatic stress on viscoelastic Rayleigh wave propagation. Alam et al. [14,15] presented the outcomes of hydrostatic stress on Rayleigh and Love-type wave propagations.

The magneto-elastic materials exhibit the magneto and elastic properties. The Earth's layer consists of different kinds of sedimentary, volcanic, and metamorphic rocks. Some ferromagnetic minerals such as nickel, iron, and cobalt have the ability to generate magnetic fields. Rayleigh wave propagation in magneto-elastic materials is entirely interesting due to its various applications and properties in different fields, including geophysics, geo-tectonics, seismology, astrophysics, acoustic, defectoscopy, and optics. Said [16], Majhi et al. [17], Shaw et al. [18], and Sahu et al. [19] carried out some research works in this field.

In the present paper, Stoneley wave propagation was investigated through the corrugated irregular common interface of two dissimilar Magneto-elastic Transversely Isotropic (MTI) half-space media. In Section 2, basic equations are elaborated via Lame's potential method. To find a general solution, those equations are enlightened. In Sections 4, 5, and 6, particular solutions are suggested. These solutions at the corrugated joint between two dissimilar media with the suitable boundary condition to obtain the dispersion relation of Stoneley waves in Magneto-elastic Transversely Isotropic (MTI) media. In Section 3, We formulate the problem and in Section 7, we get the desired dispersion relation. The outcomes are numerically computed and graphically illuminated in Section 8. Inferred observations are given in the last section.

\section{Basic equations}

The equations of motion, introduced by Biot, for a transversely isotropic elastic half-space with magnetic effect and hydrostatic state stress are given in the following [20]:

$$
\tau_{i j, j}+(\vec{J} \times \vec{B})_{i}-p_{i} \nabla^{2} u_{i}=\rho_{i} \frac{\partial^{2} u_{i}}{\partial t^{2}}
$$

Given that the assumed half space is a perfect electric conductor, Maxwell's equations in the linear form were taken into account for the electromagnetic field in the absence of displacement current [21]:

$$
\operatorname{curl} \vec{h}=\vec{j}, \quad \operatorname{curl} \vec{E}=-\mu_{e} \frac{\partial \vec{h}}{\partial t}, \quad \operatorname{div} \vec{h}=0,
$$

where:

$$
\vec{h}=\operatorname{curl}\left(\vec{u} \times \vec{H}_{0}\right),
$$

and:

$$
\vec{H}=\vec{H}_{0}+h(x, z, t)
$$

Here, it is assumed that MTI half-space is under constant $H_{0}$ acts on $y$ axis.

This wave is polarized in the $x z$-plane. Therefore, the values of $u$ and $w$ are not zero, but $v=0$. Moreover, $u$ and $w$ are independent of $y$, i.e.:

$$
u=u(x, z, t), \quad w=w(x, z, t), \quad v=0,
$$

and:

$$
\frac{\partial}{\partial y} \equiv 0
$$

Substituting Eqs. (1) and (4) into the two-dimensional form, we have:

$$
\frac{\partial \tau_{x x}}{\partial x}+\frac{\partial \tau_{x z}}{\partial z}-p_{i} \nabla^{2} u+F_{1}=\rho_{i} \frac{\partial^{2} u}{\partial t^{2}}
$$

and:

$$
\frac{\partial \tau_{z x}}{\partial x}+\frac{\partial \tau_{z z}}{\partial z}-p_{i} \nabla^{2} w+F_{3}=\rho_{i} \frac{\partial^{2} w}{\partial t^{2}},
$$

where $F_{i}=(J \times B)_{i}$.

Non-vanishing stress components of transversely isotropic media in $x z$-plane are [22]: 


$$
\begin{aligned}
\tau_{x x} & =c_{11} \frac{\partial u}{\partial x}+c_{13} \frac{\partial w}{\partial z}, \quad \tau_{z x}=c_{44}\left(\frac{\partial u}{\partial z}+\frac{\partial w}{\partial x}\right) \\
\tau_{z z} & =c_{13} \frac{\partial u}{\partial x}+c_{33} \frac{\partial w}{\partial z} .
\end{aligned}
$$

Substituting Eqs. (2), (3), and (7) into Eqs. (5) and (6), we obtain:

$$
\begin{aligned}
&\left(c_{11}\right.\left.-p_{i}+2 \mu_{e} H_{0}^{2}\right) \frac{\partial^{2} u}{\partial x^{2}}+\left(c_{13}+c_{44}+2 \mu_{e} H_{0}^{2}\right) \frac{\partial^{2} w}{\partial x \partial z} \\
&+\left(c_{44}-p_{i}\right) \frac{\partial^{2} u}{\partial z^{2}}=\rho_{i} \frac{\partial^{2} u}{\partial t^{2}}
\end{aligned}
$$

and:

$$
\begin{gathered}
\left(c_{44}-p_{i}\right) \frac{\partial^{2} w}{\partial x^{2}}+\left(c_{13}+c_{44}+2 \mu_{e} H_{0}^{2}\right) \frac{\partial^{2} u}{\partial x \partial z} \\
+\left(c_{33}-p_{i}+2 \mu_{e} H_{0}^{2}\right) \frac{\partial^{2} w}{\partial z^{2}}=\rho_{i} \frac{\partial^{2} w}{\partial t^{2}} .
\end{gathered}
$$

\section{Formulation of the problem}

In order to study Stoneley wave, a mathematical model shown in Figure 1 was employed which consisted of transversely magneto elastic isotropic semi-infinite media under hydrostatic stress $M_{1}:-\infty \leq z \leq \xi(x)$ and $M_{2}: \xi(x) \leq z \leq \infty$ with different elastic properties and corrugation at interface between two semi-infinite media, as shown earlier. This Cartesian system is usually selected where the $x$-axis is parallel to the wave traversal and $z$-axis is vertically downwards. $\xi(x)$ is a periodic and continuous function of $x$. To exhibit the common surface as represented in Figure 1, the suitable Fourier series expansion for the function was suggested by Singh [10]:

$$
\xi(x)=\sum_{n=1}^{\infty}\left(\xi_{n} e^{i n \alpha x}+\xi_{-n} e^{-i n \alpha x}\right),
$$

where $\xi_{n}$ and $\xi_{-n}$ are the coefficients of the $n$ th-order Fourier series expansion such that we have:

$$
\xi_{ \pm n}= \begin{cases}\frac{a}{2}, & \text { for } n=1 \\ \frac{A_{n}+B_{n}}{2}, & \text { for } n=2,3,4 \cdots\end{cases}
$$

Through the aforementioned $\xi_{n}$ and $\xi_{-n}, \xi(x)$ was given by Singh as follows [10]:

$$
\xi(x)=a \cos (\alpha x)+\sum_{n=2}^{\infty}\left[A_{n} \cos (n \alpha x)+B_{n} \sin (n \alpha x)\right] .
$$

\section{Solution of the upper half-space}

According to Helmholtz's theorem, the displacement vector $\vec{u}$ can be inscribed in terms of the displacement potentials $\varphi$ and $\psi$, given by:

$$
\vec{u}=\vec{\nabla} \varphi+\vec{\nabla} \times \psi .
$$

Eq. (10) reduces to:

$$
u_{1}(x, z, t)=\frac{\partial \varphi_{1}}{\partial x}-\frac{\partial \psi_{1}}{\partial z}
$$

and:

$$
w_{1}(x, z, t)=\frac{\partial \varphi_{1}}{\partial z}+\frac{\partial \psi_{1}}{\partial x} .
$$

Substituting Eq. (11) into Eqs. (8) and (9), we get:

$$
\begin{aligned}
& \left(c_{11}-p_{1}+2 \mu_{e} H_{0}^{2}\right) \frac{\partial^{2} \varphi_{1}}{\partial x^{2}}+\left(c_{13}+2 c_{44}-p_{1}\right. \\
& \left.\quad+2 \mu_{e} H_{0}^{2}\right) \frac{\partial^{2} \varphi_{1}}{\partial z^{2}}=\rho_{1} \frac{\partial^{2} \varphi_{1}}{\partial t^{2}}, \\
& \left(c_{11}-c_{13}-c_{44}-p_{1}\right) \frac{\partial^{2} \psi_{1}}{\partial x^{2}}+\left(c_{44}-p_{1}\right) \frac{\partial^{2} \psi_{1}}{\partial z^{2}} \\
& =\rho_{1} \frac{\partial^{2} \psi_{1}}{\partial t^{2}},
\end{aligned}
$$

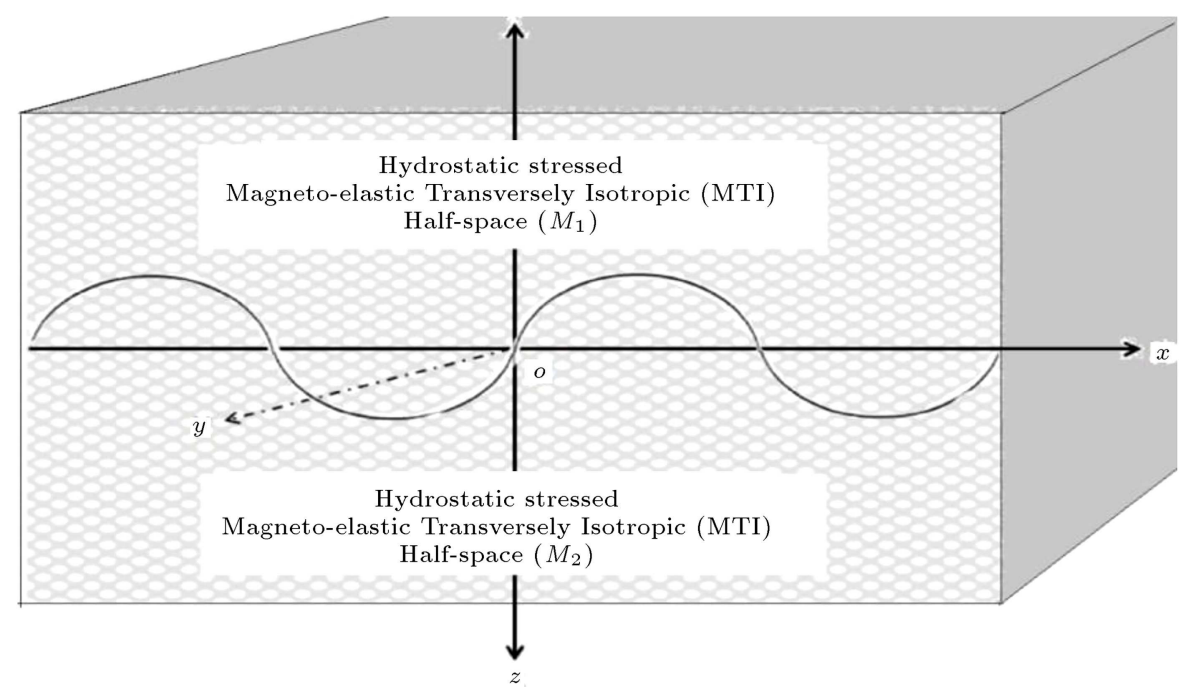

Figure 1. Geometry of the problem. 


$$
\begin{aligned}
& \left(c_{44}-p_{1}\right) \frac{\partial^{2} \psi_{1}}{\partial x^{2}}+\left(c_{33}-c_{13}-c_{44}-p_{1}\right) \frac{\partial^{2} \psi_{1}}{\partial z^{2}} \\
& =\rho_{1} \frac{\partial^{2} \psi_{1}}{\partial t^{2}} \\
& \left(c_{13}+2 c_{44}-p_{1}+2 \mu_{e} H_{0}^{2}\right) \frac{\partial^{2} \varphi_{1}}{\partial x^{2}}+\left(c_{33}+2 \mu_{e} H_{0}^{2}\right. \\
& \left.-p_{1}\right) \frac{\partial^{2} \varphi_{1}}{\partial z^{2}}=\rho_{1} \frac{\partial^{2} \varphi_{1}}{\partial t^{2}} .
\end{aligned}
$$

While Eqs. (12) and (15) express the compressive wave associated with $x$ and $z$ directions, respectively, Eqs. (13) and (14) express the shear wave associated with $x$ and $z$ directions, respectively. Undoubtedly, the body wave velocity is different in $x$ and $z$ directions. Since the present study is primarily focused on the Stoneley wave propagation only in the $x$ direction, Eqs. (12) and (14) have been taken into account.

For the harmonic travelling wave that propagates along the $x$ direction, we pursued an analytical solution to Eqs. (12) and (14) in the following form:

$$
\left[\varphi_{1}, \psi_{1}\right](x, z, t)=\left[\varphi_{1}(z), \psi_{1}(z)\right] \exp [i k(x-c t)]
$$

Substituting Eq. (16) into Eqs. (12) and (14), we obtain:

$$
\left(D^{2}-s_{1}^{2}\right) \varphi_{1}(z)=0
$$

and:

$$
\left(D^{2}-s_{2}^{2}\right) \psi_{1}(z)=0
$$

where $D^{2}=\frac{d^{2}}{d z^{2}}$ and $s_{1}$ and $s_{2}$ are described in Appendix.

Therefore, the solutions to Eqs. (17) and (18) are given as follows:

$$
\varphi_{1}(z)=A_{1} \exp \left(s_{1} z\right)+B_{1} \exp \left(-s_{1} z\right)
$$

and:

$$
\psi_{1}(z)=C_{1} \exp \left(s_{2} z\right)+D_{1} \exp \left(-s_{2} z\right) .
$$

Thus, the potential functions $\varphi_{1}$ and $\psi_{1}$ are given as:

$$
\begin{aligned}
\varphi_{1}(x, z, t)= & \left\{A_{1} \exp \left(s_{1} z\right)+B_{1} \exp \left(-s_{1} z\right)\right\} \exp \\
& {[i k(x-c t)] }
\end{aligned}
$$

and:

$$
\begin{aligned}
\psi_{1}(x, z, t)= & \left\{C_{1} \exp \left(s_{2} z\right)+D_{1} \exp \left(-s_{2} z\right)\right\} \exp \\
& {[i k(x-c t)] }
\end{aligned}
$$

For the upper half-space, we have:

$$
\varphi_{1}(x, z, t)=A_{1} \exp \left(s_{1} z\right) \exp [i k(x-c t)]
$$

and:

$$
\psi_{1}(x, z, t)=C_{1} \exp \left(s_{2} z\right) \exp [i k(x-c t)] .
$$

For the upper half-space, the solutions are given by:

$$
\begin{aligned}
u_{1}(x, z, t)= & {\left[i k A_{1} \exp \left(s_{1} z\right)-s_{2} C_{1} \exp \left(s_{2} z\right)\right] \exp } \\
& {[i k(x-c t)] }
\end{aligned}
$$

and:

$$
\begin{aligned}
w_{1}(x, z, t)= & {\left[s_{1} A_{1} \exp \left(s_{1} z\right)+i k C_{1} \exp \left(s_{2} z\right)\right] \exp } \\
& {[i k(x-c t)] . }
\end{aligned}
$$

\section{Solution of the lower half-space}

By solving the lower half-space similarly, we have the potential function as shown in the following:

$$
\begin{aligned}
\varphi_{2}(x, z, t)= & \left\{A_{2} \exp \left(r_{1} z\right)+B_{2} \exp \left(-r_{1} z\right)\right\} \exp \\
& {[i k(x-c t)], }
\end{aligned}
$$

and:

$$
\begin{aligned}
\psi_{2}(x, z, t)= & \left\{C_{2} \exp \left(r_{2} z\right)+D_{2} \exp \left(-r_{2} z\right)\right\} \exp \\
& {[i k(x-c t)], }
\end{aligned}
$$

where $r_{1}, r_{2}$ are given in Appendix.

For the lower half-space, we have:

$$
\varphi_{2}(x, z, t)=B_{2} \exp \left(-r_{1} z\right) \exp [i k(x-c t)],
$$

and:

$$
\psi_{2}(x, z, t)=D_{2} \exp \left(-r_{2} z\right) \exp [i k(x-c t)] .
$$

The solutions for the lower half-space are given by:

$$
\begin{aligned}
u_{2}(x, z, t)= & {\left[i k B_{2} \exp \left(-r_{1} z\right)+r_{2} D_{2} \exp \left(-r_{2} z\right)\right] } \\
& \exp [i k(x-c t)]
\end{aligned}
$$

and:

$$
\begin{aligned}
w_{2}(x, z, t)= & {\left[-r_{1} B_{2} \exp \left(-r_{1} z\right)+i k D_{2} \exp \left(-r_{2} z\right)\right] } \\
& \exp [i k(x-c t)] .
\end{aligned}
$$

\section{Boundary conditions}

The suitable boundary conditions must be satisfied at the common corrugated interface (i.e., at $z=\xi(x)=$ $a \cos (\alpha x))$ :

a. Displacement components are continuous:

$$
u_{1}=u_{2}, \quad \text { and } \quad w_{1}=w_{2} .
$$

b. Stresses are continuous:

$$
\text { i. } \begin{aligned}
\left(\tau_{z z}^{1}\right. & \left.+\tau_{z z}^{M_{1}}\right)-\xi^{\prime}(x)\left(\tau_{z x}^{1}+\tau_{z x}^{M_{1}}\right) \\
& =\left(\tau_{z z}^{2}+\tau_{z z}^{M_{2}}\right)-\xi^{\prime}(x)\left(\tau_{z x}^{2}+\tau_{z x}^{M_{2}}\right),
\end{aligned}
$$

ii. $\left(\tau_{x z}^{1}+\tau_{x z}^{M_{1}}\right)-\xi^{\prime}(x)\left(\tau_{x x}^{1}+\tau_{x x}^{M_{1}}\right)$

$$
=\left(\tau_{x z}^{2}+\tau_{x z}^{M_{2}}\right)-\xi^{\prime}(x)\left(\tau_{x x}^{2}+\tau_{x x}^{M_{2}}\right) .
$$




\section{Dispersion relation}

By substituting the values of $u_{1}, w_{1}, u_{2}$, and $w_{2}$ into the aforementioned Boundary Conditions (BCs), the following homogeneous algebraic system of equations for unknowns $A_{1}, B_{2}, C_{1}$, and $D_{2}$ would be achieved. This algebraic system of equations can be expressed by the matrix-vector term as follows:

$$
Z_{4 \times 4} Y_{4 \times 1}=\mathbf{0}
$$

where $Z_{4 \times 4}$ is a four-by-four matrix given by $Z_{4 \times 4}=\left[\alpha_{i j}\right],(i, j=1,2, \cdots, 6)$ and $Y_{4 \times 1}$ is a four-by-one column matrix described by $Y_{4 \times 1}=$ $\left[\begin{array}{llll}A_{1} & B_{2} & C_{1} & D_{2}\end{array}\right]^{T}$. In addition, the values for $\alpha_{i j}$ are given in Appendix.

To achieve a non-trivial solution to the aforementioned homogeneous system of equations, the determinant of the given matrix should be zero, i.e., $\left|\alpha_{i j}\right|=0$; this gives the required dispersion relation of Stoneley waves in a presumed structure.

\section{Numerical conclusion and discussion}

An interpretive inspection was made to emphasize the effects of various elastic parameters against the velocity of Stoneley wave propagation. The dispersion of Eq. (33) was used for numerical interpretation and graphical depiction.

i. For the upper half-space $M_{1}$, take the example of Magnesium material [23] into account:

$$
\begin{aligned}
& c_{11}=0.597 \times 10^{11} \mathrm{~N} / \mathrm{m}^{2}, \\
& c_{13}=0.217 \times 10^{11} \mathrm{~N} / \mathrm{m}^{2}, \\
& c_{33}=0.617 \times 10^{11} \mathrm{~N} / \mathrm{m}^{2}, \\
& c_{44}=0.164 \times 10^{11} \mathrm{~N} / \mathrm{m}^{2},
\end{aligned}
$$

and:

$$
\rho_{1}=1740 \mathrm{~kg} / \mathrm{m}^{3} \text {. }
$$

ii. For the lower half-space $M_{2}$, take the example of Beryllium material [24] into account:

$$
\begin{aligned}
& \tilde{c}_{11}=2.293 \times 10^{11} \mathrm{~N} / \mathrm{m}^{2}, \\
& \tilde{c}_{13}=0.14 \times 10^{11} \mathrm{~N} / \mathrm{m}^{2}, \\
& \tilde{c}_{33}=3.364 \times 10^{11} \mathrm{~N} / \mathrm{m}^{2}, \\
& \tilde{c}_{44}=1.625 \times 10^{11} \mathrm{~N} / \mathrm{m}^{2},
\end{aligned}
$$

and:

$$
\rho_{2}=1040 \mathrm{~kg} / \mathrm{m}^{3} \text {. }
$$

The present study aimed to examine the consequences of magneto-elastic coupling parameter for both upper and lower half-spaces $\left(m_{H}=\frac{\mu_{e} H_{0}^{2}}{c_{11}}, l_{H}=\frac{\tilde{\mu}_{e} \tilde{H}_{0}^{2}}{\tilde{c}_{11}}\right)$ of hydrostatic stress for the upper and lower half-spaces $\left(P_{1}=\frac{p_{1}}{c_{11}}, P_{2}=\frac{p_{2}}{\tilde{c}_{11}}\right)$ and position parameter $(t=$ $\left.\frac{x}{h}\right)$ for the proposed model, respectively. To this end, dimensionless phase velocity $\left(c / c_{1}\right)$ against the dimensionless common surface corrugation parameter $(a \alpha)$ is plotted for each of Figures 2-6 for their different values. In every figure, the vertical axis shows the

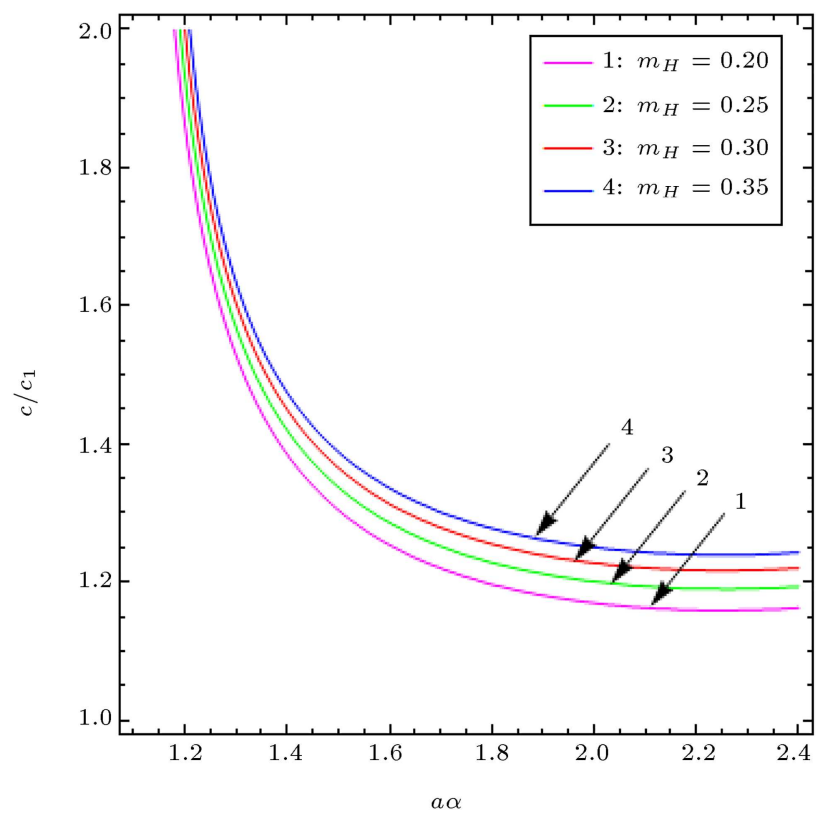

Figure 2. Influence of Stoneley wave phase velocity $\left(c / c_{1}\right)$ at increasing values of $m_{H}$ against corrugation parameter $(a \alpha)$.

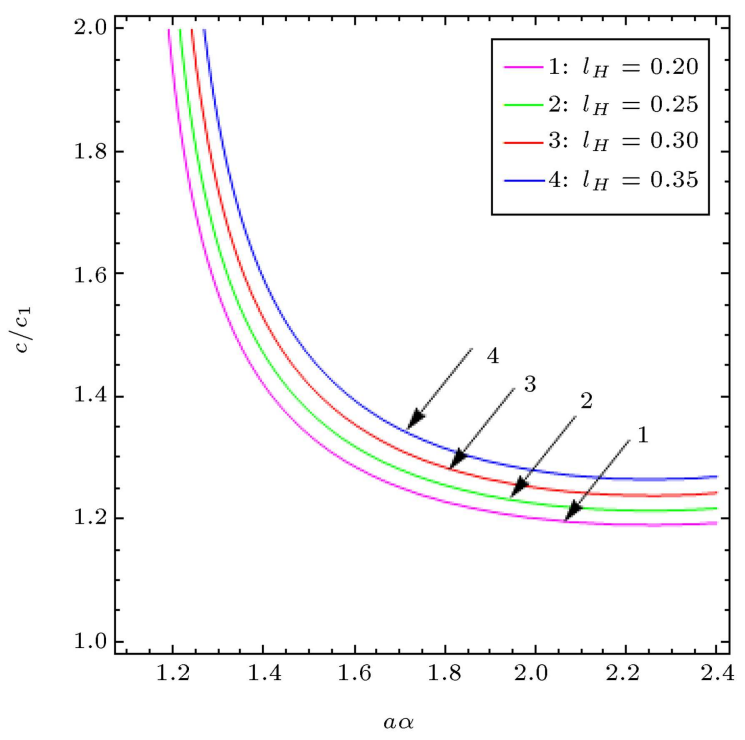

Figure 3. Influence of Stoneley wave phase velocity $\left(c / c_{1}\right)$ at different values of $l_{H}$ against corrugation parameter $(a \alpha)$. 


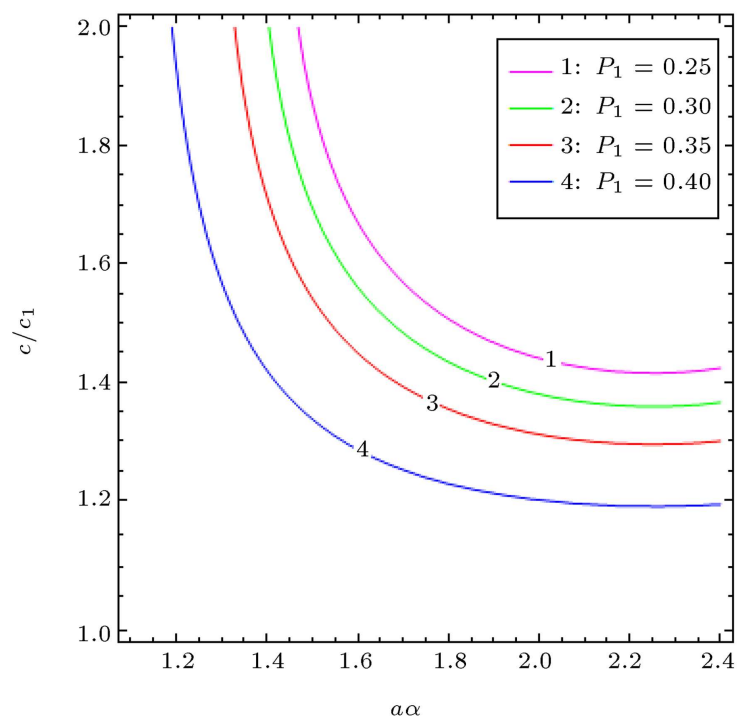

Figure 4. Stoneley wave phase velocity $\left(c / c_{1}\right)$ at different values of $P_{1}$ against corrugation parameter $(a \alpha)$.

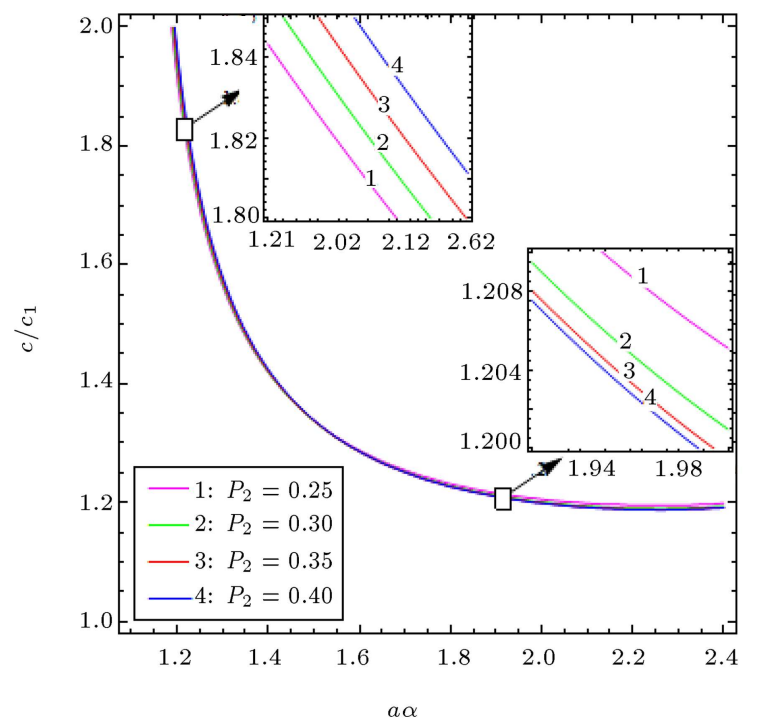

Figure 5. Stoneley wave phase velocity $\left(c / c_{1}\right)$ at increasing values of $P_{2}$ against corrugation parameter $(a \alpha)$.

dimensionless phase velocity $\left(c / c_{1}\right)$ and the horizontal axis presents the dimensionless common surface corrugation parameter $(a \alpha)$.

Figure 2 presents the effect of $m_{H}$ on the Stoneley waves phase velocity. It also shows the fluctuation in the phase velocity of Stoneley wave against the dimensionless common surface corrugation parameter at different values of $m_{H}$. According to different values of $m_{H}$ and the corresponding curves, upon increasing the value of $m_{H}$, the phase velocity of the Stoneley wave in Magnesium material would increase.

Figure 3 demonstrates some curves that are plotted for dimensionless phase velocity against the dimensionless common surface corrugation parameter at

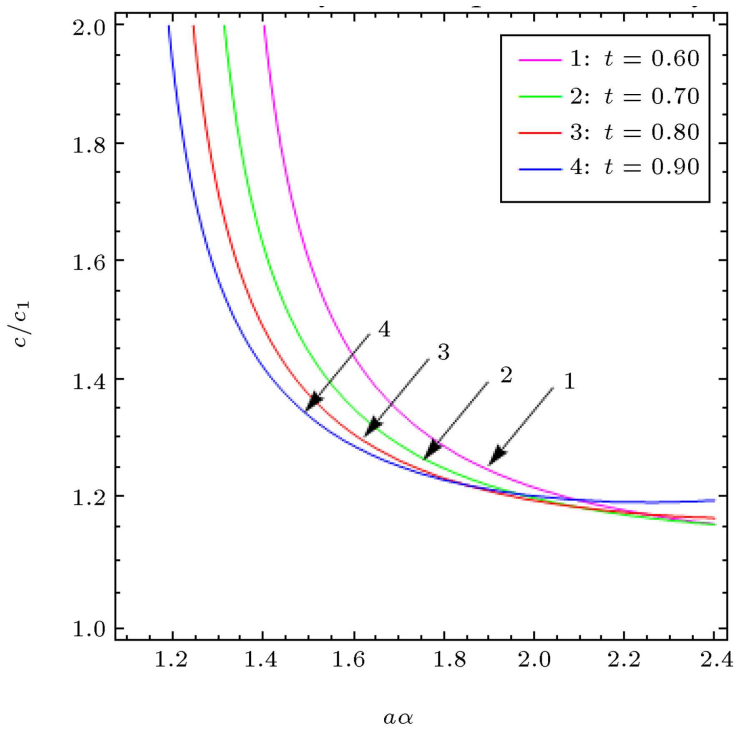

Figure 6. Stoneley wave phase velocity $\left(c / c_{1}\right)$ at different values of position parameter $(t)$ against corrugation parameter $(a \alpha)$.

different values of magneto-elastic coupling parameter $\left(l_{H}\right)$ in the lower half-space. As observed in this set of curves $(1,2,3,4)$, the magneto-elastic parameter $\left(l_{H}\right)$ has proportional impacts on the phase velocity in Beryllium material.

Figure 4 shows the effect of hydrostatic stress parameter $\left(P_{1}\right)$. According to this figure, for a particular dimensionless corrugation parameter, the hydrostatic stress parameter has unfavorable effects on the dimensionless phase velocity in Magnesium material.

Figure 5 elaborates the effect of hydrostatic stress parameter $\left(P_{2}\right)$ associated with the lower half-space. As observed, while the phase velocity increased uniformly for the frequency region $a \alpha<1.3$, it decreased in the frequency region $a \alpha>1.3$ upon increasing the magnitude of the hydrostatic stress parameter.

Figure 6 depicts the dispersion curves of Stoneley wave in case the position parameter $(t)$ is taken into account for the variation. A meticulous observation of this figure reveals that the phase velocity follows a decreasing trend.

Figure 7 shows the correlative study of dispersion curves in different cases. Curve 1 shows a case when the upper half-space is free from magnetic field $\left(m_{H}=0\right)$. Curve 2 implicates a case when the lower half-space is free from magnetic field $\left(l_{H}=0\right)$. Curve 3 refers to a case when the upper half-space is free from hydrostatic state of stress $\left(P_{1}=0\right)$. Curve 4 is a case when the lower half-space is free from hydrostatic state of stress $\left(P_{2}=0\right)$. As observed, Curve $3\left(m_{H}=0\right)$ and Curve 1 $\left(P_{1}=0\right)$ support higher and lower phase velocities, respectively, than all other cases.

Given that the phase velocity $\left(c / c_{1}\right)$ is dependent 


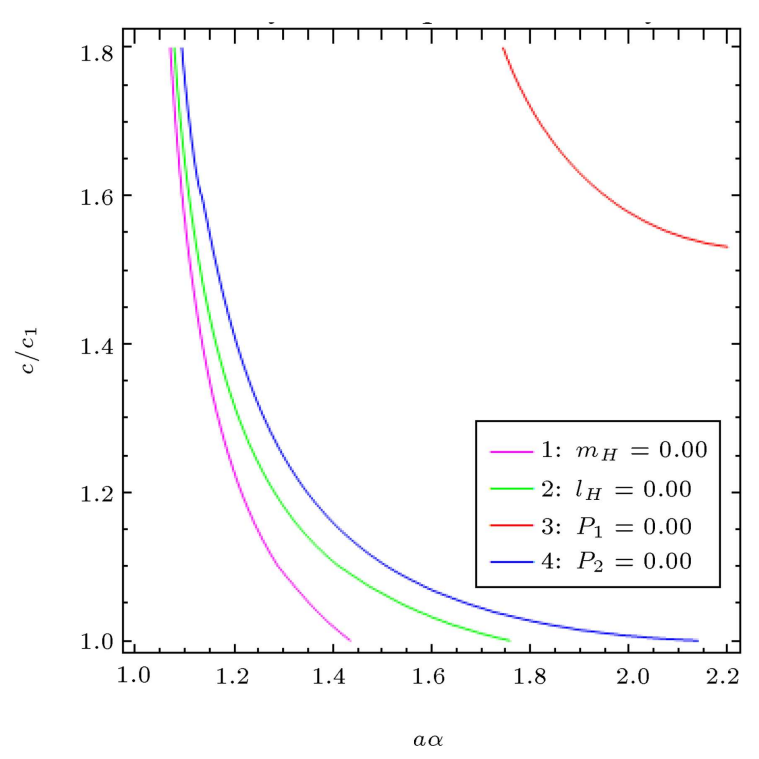

Figure 7. Stoneley wave phase velocity $\left(c / c_{1}\right)$ in the absence of different parameters against corrugation parameter $(a \alpha)$.

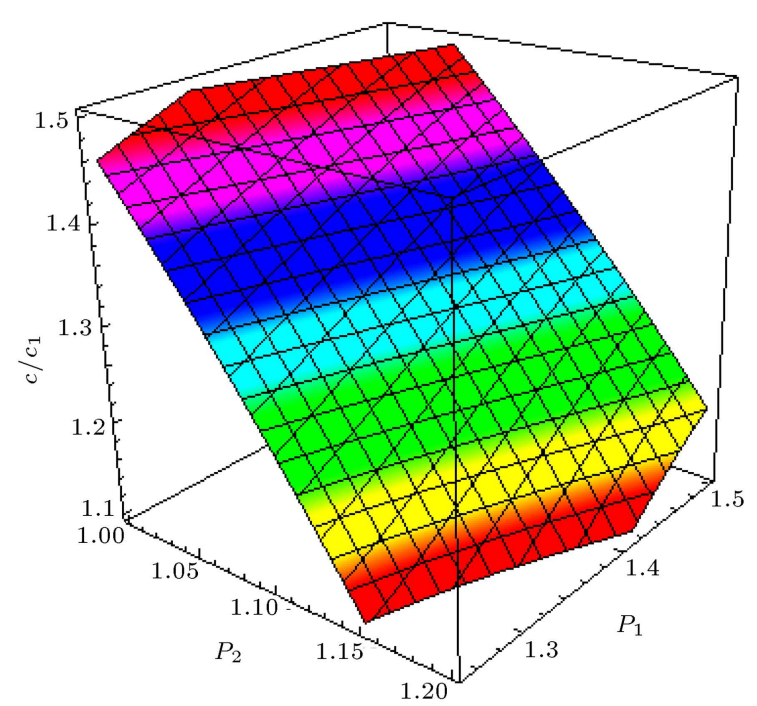

Figure 8. Variation of Stoneley wave velocity $\left(c / c_{1}\right)$ with respect to $P_{1}$ and $P_{2}$.

on the magneto-elasticity and hydrostatic stresses, the surface plot of phase velocity against varying hydrostatic stress parameters $\left(P_{1}\right.$ and $\left.P_{2}\right)$ and magnetoelasticity parameters $\left(m_{H}\right.$ and $\left.l_{H}\right)$ are shown in Figures 8 and 9 , respectively.

\section{Conclusions}

In this study, an analytical approach was employed to examine the Stoneley wave propagation through the corrugated interface of two Magneto-elastic Transversely Isotropic (MTI) half-spaces. A dispersion equation that could measure the velocity of the Stoneley waves was obtained. All the wave fronts were

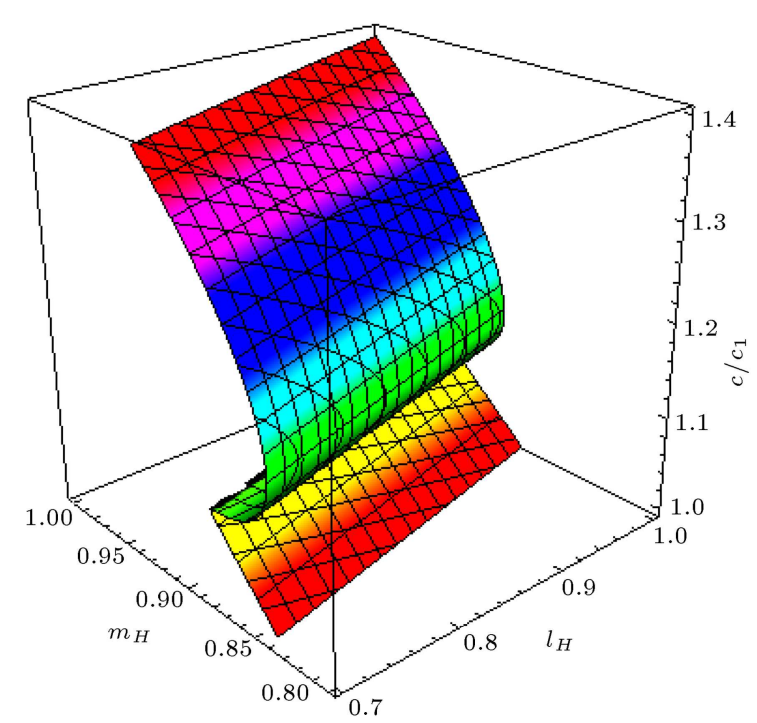

Figure 9. Variation in Stoneley wave velocity $\left(c / c_{1}\right)$ with respect to $m_{H}$ and $l_{H}$.

significantly exerted by different parameters and were graphically depicted using $2 \mathrm{D}$ and $3 \mathrm{D}$ plots. Based on the theoretical and numerical studies, it can be concluded that:

1. The magneto-elastic coupling parameters for both half-spaces followed an increasing trend in terms of the phase velocity. The phase velocity was affected the most by the absence of magneto-elastic parameter in the upper half-space and the least by the absence of the magneto-elastic coupling parameter;

2. Although the hydrostatic stress parameter for the upper half-space had inverse effect on the phase velocity, for the lower half-space, it had a mixed variation in the phase velocity.

\section{Nomenclature}

$\begin{array}{ll}\tau_{i j} & \text { Component of stress vector } \\ p_{i} & \text { Hydrostatic stress } \\ \rho_{i} & \text { Mass density } \\ c & \text { Common wave velocity } \\ k & \text { Wave number } \\ \vec{J} & \text { Electric current density } \\ j & \text { Electric intensity vector } \\ \vec{B} & \text { Magnetic induction vector } \\ F_{i} & \text { Components of Lorentz's force in the } \\ & i \text { th direction } \\ u_{i} & \text { Displacement component } \\ \vec{E} & \text { Electric intensity vector } \\ \vec{H} & \text { Magnetic field vector }\end{array}$




\begin{tabular}{|c|c|}
\hline$H_{0}, \tilde{H}_{0}$ & $\begin{array}{l}\text { Primary magnetic field of upper and } \\
\text { lower half-spaces, respectively }\end{array}$ \\
\hline$\vec{h}$ & Perturbed magnetic field vector \\
\hline$\mu_{e}, \tilde{\mu}_{e}$ & $\begin{array}{l}\text { Magnetic permeability of upper and } \\
\text { lower half-space respectively }\end{array}$ \\
\hline$c_{i j}, \tilde{c}_{i j}$ & $\begin{array}{l}\text { Elastic constants of upper and lower } \\
\text { half-space respectively }\end{array}$ \\
\hline$c_{i}$ & Wave velocity of respective layers \\
\hline$A_{n}$ & $\begin{array}{l}\text { Cosine coefficients of Fourier series } \\
\text { expansion of order } n \text { respectively }\end{array}$ \\
\hline$B_{n}$ & $\begin{array}{l}\text { Sine coefficients of } n \text {th order Fourier } \\
\text { series expansion }\end{array}$ \\
\hline$a$ & Amplitude of the corrugated surface \\
\hline$\alpha$ & Wave number of corrugations \\
\hline$\varphi, \psi$ & Lame's potentials \\
\hline$m_{H}, l_{H}$ & $\begin{array}{l}\text { Dimensionless magnetic coupling } \\
\text { parameter of upper and lower } \\
\text { half-spaces, respectively }\end{array}$ \\
\hline
\end{tabular}

\section{References}

1. Stoneley, R. "Elastic waves at the surface of separation of two solids", Proc. of Royal Soc. Lond., 106, pp. 416428 (1924).

2. Ashour, A.S. "Theoretical investigation of Stoneley wave attenuation and dispersion in a fluid filled fracture in transversely isotropic formation", ARI- An Int. J. for Physics and Eng. Sci., 51, pp. 254-257 (1999).

3. Abo-Dahab, S.M. "Propagation of Stoneley waves in magneto-thermoelastic materials with voids and two relaxation times", J. of Vib. and Cont., 21, pp. 11441153 (2015).

4. Tiwana, M.H., Ahmed, S., Mann, A.B., and Naqvi, Q.A. "Point source diffraction from a semi-infinite perfect electromagnetic conductor half plane", Optik, 135, pp. 1-7 (2017).

5. Sanjeev, S.A., Saroj, P.K., and Paswan, B. "Shear waves in a heterogeneous fiber-reinforced layer over a half-space under gravity", Int. J. of Geomech., 15(4), pp. 014-048 (2014).

6. Alam, P., Kundu, S., and Gupta, S. "Love-type wave propagation in a hydrostatic stressed magneto-elastic transversely isotropic strip over an inhomogeneous substrate caused by a disturbance point source", $J$. of Intl. Material Sys. and Struc., 29, pp. 2508-2521 (2018).

7. Kundu, S., Alam, P., and Gupta, S. "Shear waves in magneto-elastic transversely isotropic (MTI) layer bonded between two heterogeneous elastic media", Mech. of Adv. Materials and Struc., 26, pp. 407-415 (2019).
8. Singh, B. "Wave propagation in a rotating transversely isotropic two-temperature generalized thermoelastic medium without dissipation", Int. J. of Therm. (2016). In Press, DOI: 10.1007/s10765-015-2015-z

9. Vishwakarma, S.K. and Xu, R. "Rayleigh wave dispersion in an irregular sandy Earth's crust over orthotropic mantle", Appl. Math. Modelling, 40, pp. 8647-8659 (2016).

10. Singh, S.S. "Love wave at a layer medium bounded by irregular boundary surfaces", J. of Vib. and Cont., 17, pp. 789-795 (2011).

11. Alam, P., Kundu, S., and Gupta, S. "Dispersion study of SH-wave propagation in an irregular magneto-elastic anisotropic crustal layer over an irregular heterogeneous half-space", J. of King Saud University-Sci., 30(3), pp. 301-310 (2016).

12. Saroj, P.K., Sanjeev, S.A., and Chattopadhyay, A. "Dynamic response of corrugation and rigid boundary surface on Love-type wave propagation in orthotropic layered medium", J. of Porous Media, 21, pp. 11631176 (2018).

13. Addy, S.K. and Chakraborty, N.R. "Rayleigh waves in a viscoelastic half-space under initial hydrostatic stress in presence of the temperature field", Int. J. of Math. and Math. Sci., 24, pp. 3883-3894 (2005).

14. Alam, P., Kundu, S., and Gupta, S. "Effect of magneto-elasticity, hydrostatic stress and gravity on Rayleigh waves in a hydrostatic stressed magnetoelastic crystalline medium over a gravitating half-space with sliding contact", Mech. Research Comm., 89, pp. 11-17 (2018).

15. Alam, P., Kundu, S., and Gupta, S. "Dispersion and attenuation of Love-type waves due to a point source in magneto-viscoelastic layer", J. of Mech., 34, pp. 801-816 (2018).

16. Said, S.M. "Influence of gravity on generalized magnetothermoelastic medium for three-phase-lag model", $J$. of Comput. Appl. Math., 291, pp. 142-157 (2016).

17. Majhi, S., Pal, P.C., and Kumar, S. "Reflection and transmission of plane SH-waves in an initially stressed inhomogeneous anisotropic dispersion study of SH-wave propagation magnetoelastic medium", $J$. of Seism., 21, pp. 155-163 (2016).

18. Shaw, S., Biswas, S., and Mukhopadhyay, B. "Rayleigh waves in a thermoorthotropic medium: A dynamic analysis", Comput. Therm. Sci.: An Int. J., 10, pp. $557-574$ (2018).

19. Sahu, S.A., Chaudhary, S., Saroj, P.K., and Chattopadhyay, A. "Rayleigh waves in liquid layer resting over an initially stressed orthotropic half-space under self-weight", Arab. J. of Geosci. (2017). In Press DOI: $10.1007 / \mathrm{s} 12517-017-2924-1$ 
20. Biot, M.A., Mechanics of Incremental Deformations, John Wiley \& Sons, Inc., New York (1965).

21. Mukhopadhyay, S. "Effects of thermal relaxations on thermoviscoelastic interactions in an unbounded body with a spherical cavity subjected to a periodic loading on the boundary", J. of Therm. Stresses, 23, pp. 675$684(2000)$.

22. Anderson, D.L. "Elastic wave propagation in layered anisotropic Media", J. of Geophy. Research, 66, pp. 2953-2963 (1961).

23. Rehman, A., Khan, A., and Ali, A. "Rayleigh waves in a rotating transversely isotropic materials", Elect. J. Tech. Acoustics, p. 5 (2007).

24. Ding, H., Chien, W., and Zhang, I. "Elasticity of transversely isotropic materials", Springer Sci. and Business Media, 126, pp. 22-23 (2006).

\section{Appendix}

Mathematical relation between all the parameters are given below:

$$
\begin{aligned}
& c_{1}=\sqrt{\frac{c_{11}}{\rho_{1}}}, \quad s_{1}^{2}=k^{2} \frac{c_{11}-p_{1}+2 \mu_{e} H_{0}^{2}-\rho_{1} c^{2}}{c_{13}+2 c_{44}-p_{1}+2 \mu_{e} H_{0}^{2}}, \\
& r_{1}^{2}=k^{2} \frac{\tilde{c}_{11}-p_{2}+2 \tilde{\mu}_{e} \tilde{H}_{0}^{2}-\rho_{2} c^{2}}{\tilde{c}_{13}+2 \tilde{c}_{44}-p_{2}+2 \tilde{\mu}_{e} \tilde{H}_{0}^{2}}, \quad c_{2}=\sqrt{\frac{\tilde{c}_{11}}{\rho_{2}}}, \\
& s_{2}^{2}=k^{2} \frac{c_{44}-p_{1}-\rho_{1} c^{2}}{c_{33}-c_{13}-c_{44}-p_{1}}, \\
& r_{2}^{2}=k^{2} \frac{\tilde{c}_{44}-p_{2}-\rho_{2} c^{2}}{\tilde{c}_{33}-\tilde{c}_{13}-\tilde{c}_{44}-p_{2}}, \\
& \alpha_{11}=i k \exp \left(s_{1} a \cos (\alpha x)\right), \\
& \alpha_{12}=-i k \exp \left(-r_{1} a \cos (\alpha x)\right) \\
& \alpha_{13}=-s_{2} \exp \left(s_{2} a \cos (\alpha x)\right), \\
& \alpha_{14}=-r_{2} \exp \left(-r_{2} a \cos (\alpha x)\right), \\
& \alpha_{21}=s_{1} \exp \left(s_{1} a \cos (\alpha x)\right), \\
& \alpha_{22}=r_{1} \exp \left(-r_{1} a \cos (\alpha x)\right) \\
& \alpha_{23}=i k \exp \left(s_{2} a \cos (\alpha x)\right), \\
& \alpha_{24}=-i k \exp \left(-r_{2} a \cos (\alpha x)\right),
\end{aligned}
$$

$$
\begin{aligned}
& \alpha_{31}=\left[s_{1}^{2} c_{33}-k^{2} c_{13}+\mu_{e} H_{0}^{2}\left(s_{1}^{2}-k^{2}\right)\right. \\
& \left.+2 i k s_{1} a \alpha c_{44} \sin (\alpha x)\right] \exp \left(s_{1} a \cos (\alpha x)\right), \\
& \alpha_{32}=\left[\tilde{c}_{13} k^{2}-\tilde{c}_{33} r_{1}^{2}-\tilde{\mu}_{e} \tilde{H}_{0}^{2}\left(r_{1}^{2}-k^{2}\right)\right. \\
& \left.+2 i k a \alpha r_{1} \tilde{c}_{44} \sin (\alpha x)\right] \exp \left(-r_{1} a \cos (\alpha x)\right), \\
& \alpha_{33}=\left[i k s_{2}\left(c_{33}-c_{13}\right)-a \alpha c_{44}\left(s_{2}^{2}+k^{2}\right) \sin (\alpha x)\right] \\
& \exp \left(s_{2} a \cos (\alpha x)\right) \\
& \alpha_{34}=\left[i k r_{2}\left(\tilde{c}_{33}-\tilde{c}_{13}\right)+a \alpha \tilde{c}_{44}\left(r_{2}^{2}+k^{2}\right) \sin (\alpha x)\right] \\
& \exp \left(-r_{2} a \cos (\alpha x)\right) \\
& \alpha_{41}=\left[2 i k s_{1} c_{44}+a \alpha \sin (\alpha x)\left\{s_{1}^{2} c_{13}-k^{2} c_{11}\right.\right. \\
& \left.\left.+\mu_{e} H_{0}^{2}\left(k^{2}-s_{1}^{2}\right)\right\}\right] \exp \left(s_{1} a \cos (\alpha x)\right), \\
& \alpha_{42}=\left[2 i k r_{1} \tilde{c}_{44}-a \alpha \sin (\alpha x)\left\{r_{1}^{2} \tilde{c}_{13}-\tilde{c}_{11} k^{2}\right.\right. \\
& \left.\left.+\tilde{\mu}_{e} \tilde{H}_{0}^{2}\left(k^{2}-r_{1}^{2}\right)\right\}\right] \exp \left(-r_{1} a \cos (\alpha x)\right), \\
& \alpha_{43}=\left[i k a \alpha s_{2} \sin (\alpha x)\left(c_{13}-c_{11}\right)-c_{44}\left(s_{2}^{2}+k^{2}\right)\right] \\
& \exp \left(s_{2} a \cos (\alpha x)\right) \\
& \alpha_{44}=\left[\tilde{c}_{44}\left(r^{2}+k^{2}\right)-i k a \alpha r_{2} \sin (\alpha x)\left(\tilde{c}_{11}-\tilde{c}_{13}\right)\right] \\
& \exp \left(-r_{2} a \cos (\alpha x)\right) \text {. }
\end{aligned}
$$

\section{Biographies}

Soumitra Chowdhury is currently pursuing the $\mathrm{PhD}$ degree at Indian Institute of Technology (ISM) Dhanbad at the Department of Mathematics and Computing. He received his BSc degree from the Presidency College Kolkata, under the University of Calcutta in 2014 and MSc degree from IIT(ISM) Dhanbad in 2016.

Santimoy Kundu currently works as an Associate Professor at the Department of Mathematics and Computing, Indian Institute of Technology (ISM) Dhanbad, India. His research areas of interest are theoretical seismology and wave propagation aspects.

Parvez Alam currently works at the Department of Mathematics, School of Advanced Sciences, Vellore Institute of Technology, Vellore as an Assistant Professor. He completed his PhD in Applied Mathematics form IIT(ISM) Dhanbad in 2018 and MSc in Mathematics from AMU, Aligarh in 2013. His main 
research interests include theoretical seismology and solid mechanics.

Shishir Gupta is a Professor at the Department of Mathematics and Computing, Indian Institute of
Technology (ISM) Dhanbad, India. He served as the Head of the Department of the Applied Mathematics, IIT(ISM) Dhanbad from 2015 to 2018. His research areas are theoretical seismology, numerical methods and special functions. 